\title{
The interplay of sex and gender on the reactivity of the endocrine stress axis in humans Jens C Pruessner ${ }^{1,2}$
}

In response to psychological and physiological stressors, metabolic systems of the human body change their activation to increase energy availability, and allow the organism to cope with the real or perceived increase in demand. An inappropriate stress response, perhaps caused by a dysregulation of the metabolic stress response systems, is believed to be associated with an increased risk for development of (psycho) pathology. The observation that men and women differ in their stress responsivity is important in this context, as men and women also systematically differ in their risk for developing disease. Women are more likely to suffer from autoimmune disorders, anxiety disorders, dementia, to name just a few. Investigating the reasons for the stress responsivity differences between men and women has thus been an important area of research for several decades, and we are now at a point where we better understand some of the underlying mechanisms. There are both biological and cultural effects at play that contribute to establishing these reactivity differences between men and women, with recent findings providing limited support for the idea that cultural effects might have the greater impact. The current selective review summarizes some of the key findings from the last 25 years to provide an overview of this interesting topic.

\author{
Addresses \\ ${ }^{1}$ University of Constance, Department of Psychology, Constance, \\ Germany \\ ${ }^{2}$ McGill University, Department of Psychiatry, Montreal, Quebec, \\ Canada
}

Corresponding author:

Pruessner, Jens C (jens.pruessner@uni-konstanz.de)

"Every cell is sexed, every organism is gendered"

Motto of the Institute of Gender and Health of the Canadian Institutes of Health Research (CIHR)

\section{Introduction}

There is no question that men differ from women during their reproductive years in their stress reactivity. Stress reactivity is measured in different ways, including subjective perception of stress, autonomic nervous system markers like heart rate, galvanic skin response, or blood pressure, and endocrine stress markers like the human stress hormone cortisol. Especially the endocrine system has been under intense investigation, as a dysregulation of the hormonal system in charge of secreting cortisol, the hypothalamicpituitary adrenal (HPA) axis, is considered a significant risk factor for various forms of psychopathology, including depression, anxiety disorders, burnout, and psychosis [1]. Interestingly, women are more likely to be affected by these disorders than men, in a ratio that varies from 2:1 to $4: 1$, depending on the specific disorder in question $\left[2^{\bullet}\right]$.

However, the direction of these stress reactivity differences is actually inconsistent between studies, with women sometimes showing a stronger, and sometimes a weaker response, when compared to men. What factors are contributing to these differences, and the direction of these differences, has been under intense investigation for almost three decades, and has identified methodological aspects (the type of stressor used), biological (the interaction of the endocrine stress response system with other endocrine systems), and cultural (the beliefs and values that are different between men and women). In the following, these factors will briefly be reviewed, with specific examples illustrating their effects.

\section{Of stressors, hormones and culture}

One of the best known stress tests in the laboratory, the Trier Social Stress Test (TSST), consists of 5 min public speaking and $5 \mathrm{~min}$ mental arithmetic in front of an audience, with the audience typically consisting of at least two people of mixed gender. In addition, the performance is video-taped, and audio-recorded. The typical scenario is that of a mock-job interview, where the subject is asked to deliver a five minute speech to justify why he or she is the best candidate for a hypothetical job. In contrast to a real interview, there is no interaction with, or feedback from, the audience - they keep a neutral facial expression throughout, and if the subject pauses in his or her presentation, they remain silent for up to $20 \mathrm{~s}$ before asking the subject to continue to talk as long as there is still time.

After the five minute speech, the subject is asked to solve an arithmetic task - something like counting down from 
3867 in steps of 17 , with the instruction to start over when making a mistake. This laboratory test is effective in 70 to $100 \%$ of participants, with increases in the sympathetic nervous system (heart rate, blood pressure) and endocrine stress axis (ACTH, cortisol) that for cortisol range between 50 and $100 \%$ over baseline [3].

Early on, it was observed that men showed higher cortisol reactivity than women $\left[4^{\bullet}, 5^{\circ}\right]$, and the question arose where these differences were coming from. Subsequent research investigated mainly two hypotheses: One, that the differences had to do with biological differences between men and women, that is, they were a function of sex. Two, that the differences had to do with sociocultural differences between men and women, that is, they were a function of gender. For both hypotheses, supporting results could be found.

Looking into sex differences, researchers soon identified the interplay of the endocrine stress axis with another endocrine system, the hypothalamic-pituitary-gonadal (HPG) axis, as a possible mechanism with which differences could be explained. The HPG axis in women during their reproductive years predominantly stimulates production of estrogens from the gonads. Estrogens, in return, are associated with two- to three fold higher numbers of cortisol binding globulin (CBG), which as the name suggests - bind cortisol [6]. As cortisol bound to CBG can't bind to receptors, or cross the blood-brain barrier, this in effect reduces the availability of active cortisol in the system. This further explains the difference in cortisol concentration in saliva versus plasma, as only the free fraction is transferred into saliva. This is the case for both men and women as up to $95 \%$ of cortisol is bound at all times - however, in women the larger numbers of CBG might imbalance the distribution further. It is as if the women have a blunted, or temporarily impaired, stress response system, when estrogens and thus CBG levels are high.

As estrogen production and availability itself varies over the course of the menstrual cycle, soon the question emerged whether free cortisol availability would then be systematically linked to the different stages of the menstrual cycle in women. During the follicular phase, which is the first to follow the menses, the follicles in the ovaries (the female gonads) begin to grow and start to produce estrogens. Thus, during the follicular phase, which roughly lasts the first half of the menstrual cycle, estrogen levels are very low in the beginning but then continue to rise more and more until just before ovulation, they reach their highest levels. After ovulation, they remain high for a short period but if no pregnancy occurs, they drop continuously over the second half of the menstrual cycle until they are at their lowest point at the time of the menses. This picture is changed completely when women during their reproductive years are taking estrogen-containing oral contraceptives; here, estrogen levels are continuously high (even higher than during the follicular phase) during much of the menstrual cycle with the exception of the last seven days of the cycle.

Given the various levels of estrogens in these different scenarios, Kirschbaum et al. [5,7] investigated this systematically and observed that the greater the level of estrogens in circulation, the smaller was the observed endocrine response to the standardized TSST. This was quite convincing as the level of free cortisol followed a dose response of the (assumed) amounts of circulating estrogens: oral contraceptive users showed the smallest response, followed by follicular phase women, luteal phase women, and men $\left[5^{\circ}\right]$.

Progesterone, which is particularly high around ovulation and during the first half of the luteal phase, has also been shown to have dampening effects on cortisol reactivity when pharmacologically administered [8], but effects on cortisol reactivity during the menstrual cycle phase appear to be less consistent, perhaps because progesterone is also produced from the adrenals in response to stress [9].

As a side note, postmenopausal women and women who are pregnant show a very different HPG activity - in menopausal women not undergoing hormonal treatment therapy, estrogens and progesterone production has come to an almost complete halt and thus no effects on HPA axis activity are observed [10]. In contrast, pregnant women especially during the third trimester show a significantly increased activity of both the HPA and the HPG axis, having to do with fetus development, thus preventing investigations of HPG / HPA axis interaction [11].

In addition to biological factors, almost from the beginning, there was also evidence that gender and socialization played a role in the stress response-here, studies that manipulated the effects of the perception of the situation contributed to this. Again it was Kirschbaum et al. $\left[12^{\circ}\right]$ who provided the pioneering work in this area by showing that if men received social support when being exposed to psychosocial stress, their HPA axis response to the TSST was reduced as a function of the closeness to the individual providing social support. In comparing three groups (no support, stranger support, and partner support), they could show that while the stranger support diminished the cortisol response somewhat, the partner support showed the strongest decline in cortisol response when compared to the control group with no support. However, the opposite could be shown when women were stressed-here, women in the no support group showed smaller responses than the men, but these responses actually increased when they were supported by a stranger, and were strongest when their own partner 
'supported' them! Clearly, in this case psychosocial factors took the upper hand in determining the stress response - the women were perceiving the stressfulness of the situation more strongly when their partner was around.

The idea that women respond more strongly to the social aspects of a situation was properly formulated a couple of years later, when Taylor et al. [13] published a review with the title 'Tend and befriend, not fight or flight', describing the different psychological factors which make situations stressful for either men or women. Summarizing previous work from stress studies, they argued that men are more sensitive to the achievement and competitive aspects of a situation, while women respond more to the social aspects - how a situation is affecting their social standing, or their bond within an important peer group. This conclusion was partly based on work from Stroud et al. [14], who compared an achievement stressor (difficult math) with a social rejection stressor (being intentionally excluded from a group discussion), and could show that men predominantly responded to the achievement task with an increase of cortisol, while women preferentially showed the higher responses to the social rejection task.

Thus, up to this point, we have seen that both biological and sociocultural factors play a role in causing and modulating HPA axis stress responses. There have been a number of studies since, some from my own lab, who have contributed further evidence to the idea that these are important factors that determine differences between men and women. For example, when investigating the cortisol stress response in a neuroimaging environment, we developed the 'Montreal Imaging Stress 'Task' (MIST), which was loosely based on the math portion of the TSST (Dedovic et al. [15]). However, it was presented as a computer program and the algorithm to determine the math difficulty and the imposed time limit was adapting to the user's aptitude - without the user being aware of it. The goal was to make the presented tasks always a bit harder and the time constraint a bit too limited given the individual's capabilities, which creates poor performance, regardless of how hard the individual tries. While we could show robust increases in cortisol when using this task in men, we were not able to achieve similar effect sizes in women, likely owing to the fact that the task was emphasizing the achievement component of the situation. However, when we adjusted the cover story of the task, such that the experimenter was bonding with the female test subject first, and deceived her into believing that good performance was vital for the experimenter's PhD completion, women showed more robust cortisol responses than men [16].

As the final question to be addressed here, if both biological and cultural factors play a significant role, is there any evidence to suggest whether one factor plays a stronger role than the other? Here, some recent data is available where using a special design and a special population, this question could be partly answered, and it points to sociocultural factors playing the stronger role $\left[17^{\bullet \bullet}\right]$.

One of the ways that the original TSST was manipulated in order to better understand what caused its effects, was to take the audience and put it behind a one-way mirror $[18,19]$. The test subject would still be presenting in front of the panel of experts, but could no longer see it. It would be interacting with the audience via intercom, and the exact same messages would be communicated, and the exact same performance would be enforced, but without the test subjects seeing their evaluators. We had originally conceived this modification to determine what effect the amount of social-evaluative threat had on the TSST response, arguing that the threat would likely be reduced if the test subjects could no longer see the evaluation committee. We tested this modified paradigm against the standard paradigm, with groups of men and women going either through the original, or the modified paradigm. The results of this manipulation were very specific to the sex of the test subjects: While men showed no difference in terms of their stress responses regardless of whether they were seeing the panel or not [18], women showed no response when they couldn't see the panel, as compared to the standard response when they were in front of the evaluation committee [19]. In other words, when using the modified 'behind the mirror' version of the TSST, we now had a test at our disposal that maximized the differences between men and women.

In a follow-up study a number of years later, we wanted to determine what effect variations in gender identity had on the normally observed differences in stress response between men and women. To do this, we recruited from the general population individuals whose biological sex was male or female, but who were showing mismatching gender identities to various degrees - i.e., members from the LBGT community. In the most dramatic form, these were subjects who were biologically male, but whose gender identity was female (or the other way around). In reality, we measured gender identity using three different validated means, including interviews and questionnaires, and observed varying degrees of gender identity mismatch to subjects' biological sex. This approach allowed us to investigate how sex versus gender were associated with the previously observed cortisol stress response differences between men and women.

To maximize possible effects, we specifically used the 'sex-difference enhanced' version of the TSST we had developed a couple of years before [18,19]. Our hypothesis was that if the previously observed differences had more to do with biology (sex) rather than with cultural (gender) factors, then we should observe a stronger 
response in men than in women to the one-way mirror version of the TSST, regardless of gender identity. On the other hand, if it was the gender more than the sex which was determining the response differences, the responses should align according to the gender identity of the subjects, regardless of their biological sex. To test this, we created four groups of subjects: biological men who showed a male gender identity, biological men with mixed or female gender identity (members of the LBGT community who were biologically male), biological women who showed a female gender identity, and biological women who showed a mixed or male gender Identity. In the following experiment, these groups were all exposed to the modified version of the TSST, with the audience behind the one-way mirror. The results were rather unambiguous: male gender identity combined with male biological sex showed a clear response, and female gender identity combined with female biological sex failed to respond, thus replicating previous results. However, if the gender identity was more female or completely female, subjects failed to respond to our task, even if the biological sex was male. Likewise, if the gender identity was more male or completely male, subjects responded to the task even if their biological sex was female. It has to be emphasized that none of the subjects in this study had undergone sex change operations, and none were taking hormonal treatments. Thus, the results pointed to a rather stronger role of gender identity in explaining the differences between men and women, with current biological (or hormonal) factors playing a more secondary role (Figure 1). Of course, we cannot speak to the possibility that a specific hormonal milieu during critical development periods has contributed to the development of the observed variations in gender identity, thus leaving the possibility of an organizational role of biological factors. Also, we realize that it is simplistic to equate biological sex only with gonadal sex - a number of additional

Figure 1

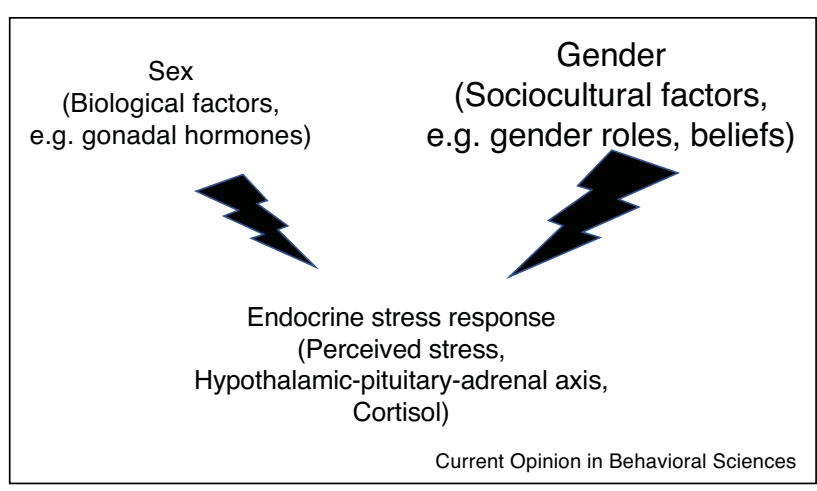

The interplay of sex (biological) and gender (sociocultural) factors on stress responsivity in men and women. At least acutely, sociocultural factors seem to play a stronger role in determining stress reactivity in both men and women. factors come into play, importantly, sensitivity for gonadal hormones, availability of enzymes to mediate hormonal effects, and at all times an interaction with environmental factors in promoting biological activity and thus influencing gender identity. We did, however, measure a wide range of hormonal factors of the HPG axis at baseline and did not observe systematic differences between the various gender identities within the same sex, suggesting that at least current biological factors were not at play.

\section{Conclusion}

Taken together, available results with respect to the effects of sex and gender on hormonal stress responses have provided evidence that both factors play a role. This could be shown across various studies and using different stress stimuli, thus suggesting that it is a stable phenomenon. The direction of these differences varies, and seems to depend on whether biological factors or cultural factors are emphasized in the study design, and the study populations. With regard to studies performed in more recent years, a picture emerges where sociocultural factors seem to play a stronger role when compared to biological ones, at least when looking comparing acute effects. This still leaves the possibility that biological factors combined with sociocultural factors shaped the stress response during critical development periods, which is difficult to speak to in cross-sectional studies. Here, longitudinal studies should investigate both hormonal and sociocultural effects throughout development to determine if and how both factors interact to shape the stress response, and eventually contribute to the risk for developing psychopathology..

\section{Conflict of interest statement}

Nothing declared.

\section{Recommended reading}

Papers of particular interest, published within the period of review, have been highlighted as:

- of special interest

$\bullet$ of outstanding interest

\section{Acknowledgments}

This work was supported by the Carl-Zeiss Stiftung and the German Scholar Organisation.

\section{References}

1. Yehuda R, Seckl J: Minireview: stress-related psychiatric disorders with low cortisol levels: a metabolic hypothesis Endocrinology 2011, 152:4496-4503 http://dx.doi.org/10.1210/ en.2011-1218.

2. Palanza P, Parmigiani S: How does sex matter? Behavior, stress - and animal models of neurobehavioral disorders. Neurosci Biobehav Rev 2017, 76:134-143 http://dx.doi.org/10.1016/j. neubiorev.2017.01.037.

Excellent review on the topic of sex differences and disease. 
3. Kirschbaum C, Pirke KM, Hellhammer DH: The Trier Social Stress Test $-\mathbf{a}$ tool for investigating psychobiological stress responses in a laboratory setting. Neuropsychobiology 1993, 28:76-81.

4. Kirschbaum C, Wüst S, Hellhammer D: Consistent sex

- differences in cortisol responses to psychological stress. Psychosom Med 1992, 54:648-657 http://dx.doi.org/10.1097/ 00006842-199211000-00004.

One of the first studies to show systematic sex differences in cortisol responses to psychological but not pharmacological manipulation.

5. Kirschbaum C, Kudielka BM, Gaab J, Schommer NC,

- Hellhammer DH: Impact of gender, menstrual cycle phase, and oral contraceptives on the activity of the hypothalamuspituitary-adrenal axis. Psychosom Med 1999, 61:154-162 http:// dx. doi.org/10.1097/00006842-199903000-00006.

Systematic study to show the effects of naturally varying and manipulated gonadal hormones on the endocrine stress response.

6. Nenke MA, Zeng A, Meyer EJ, Lewis JG, Rankin W, Johnston J, Kireta S, Jesudason S, Torpy JD: Differential effects of estrogen on corticosteroid-binding globulin forms suggests reduced cleavage in pregnancy. J Endocr Soc 2017, 1:202-210 http://dx. doi.org/10.1210/js.2016-1094.

7. Kirschbaum C, Wust S, Hellhammer DH: Consistent sex differences in cortisol responses to psychological stress. Psychosom Med 1992, 54:648-657.

8. Childs E, Dam NT, de Wit H: Effects of acute progesterone administration upon responses to acute psychosocial stress in men. Exp Clin Psychopharmacol 2010, 18:78-86.

9. Wirth MM, Meier EA, Fredrickson BL, Schultheiss OC: Relationship between salivary cortisol and progesterone levels in humans. Biol Psychol 2007, 74:104-107.

10. Lindheim SR, Legro RS, Bernstein L, Stanczyk FZ, Vijod MA Presser SC, Lobo RA: Behavioral stress responses in premenopausal and postmenopausal women and the effects of estrogen. Am J Obstet Gynecol 1992, 167:1831-1836.

11. Darne FJ, MacGarrigle HH, Lachelin GC: Diurnal variation of plasma and saliva oestrogen, progesterone, cortisol and plasma dehydroepiandrosterone sulphate in late pregnancy. Eur J Obstet Gynecol Reprod Biol 1989, 32:57-66.
12. Kirschbaum C, Klauer T, Filipp S, Hellhammer DH: Sex-specific

- effects of social support on cortisol and subjective responses to acute psychological stress. Psychosom Med 1995, 57:23-31 http://dx.doi.org/10.1097/00006842-199501000-00004.

Hallmark study to demonstrate an effect of sociocultural factors on the endocrine stress response.

13. Taylor SE, Klein LC, Lewis BP, Gruenewald TL, Gurung RA, Updegraff JA: Biobehavioral responses to stress in females: tend-and-befriend, not fight-or-flight. Psychol Rev 2000 107:411-429 http://dx.doi.org/10.1037/0033-295x.107.3.411.

14. Stroud LR, Salovey $P$, Epel ES: Sex differences in stress responses: social rejection versus achievement stress. Biol Psychiatry 2002, 52:318-327 http://dx.doi.org/10.1016/s00063223(02)01333-1.

15. Dedovic K, Renwick R, Mahani NK, Engert V, Lupien SJ, Pruessner JC: The Montreal Imaging Stress Task: using functional imaging to investigate the effects of perceiving and processing psychosocial stress in the human brain. $J$ Psychiatry Neurosci 2005, 30:319-325.

16. Duchesne A: Physiological, neural and affective responses to social evaluative stress in men and women: a question of context and menstrual cycle phases. . (PhD thesis) Montreal, Quebec, Canada: Integrated Program in Neuroscience, McGill University; 2013.

17. Juster RP, Hatzenbuehler ML, Mendrek A, Pfaus JG, Smith NG

-• Johnson PJ, Lefebvre-Louis JP, Raymond C, Marin MF, Sindi S, Lupien SJ, Pruessner JC: Sexual orientation modulates endocrine stress reactivity. Biol Psychiatry 2015, 77:668-676.

Study comparing the strength of sex versus gender effects in determining the endocrine stress response in humans, suggesting gender effects might be more dominant.

18. Andrews J, Wadiwalla M, Juster RP, Lord C, Lupien SJ, Pruessner JC: Effects of manipulating the amount of socialevaluative threat on the cortisol stress response in young healthy men. Behav Neurosci 2007, 121:871-876 http://dx.doi. org/10.1037/0735-7044.121.5.871.

19. Wadiwalla M, Andrews J, Lai B, Buss C, Lupien SJ, Pruessner JC: Effects of manipulating the amount of social-evaluative threat on the cortisol stress response in young healthy women. Stress 2010, 13:214-220 http://dx.doi.org/10.3109/ 10253890903277561 